\title{
High-throughput shadow mask printing of passive electrical components on paper by supersonic cluster beam deposition
}

Francesco Caruso, Andrea Bellacicca, and Paolo Milani

Citation: Appl. Phys. Lett. 108, 163501 (2016); doi: 10.1063/1.4947281

View online: https://doi.org/10.1063/1.4947281

View Table of Contents: http://aip.scitation.org/toc/apl/108/16

Published by the American Institute of Physics

\section{Articles you may be interested in}

Stretchable nanocomposite electrodes with tunable mechanical properties by supersonic cluster beam implantation in elastomers

Applied Physics Letters 106, 121902 (2015); 10.1063/1.4916350

Metal-polymer nanocomposite with stable plasmonic tuning under cyclic strain conditions Applied Physics Letters 107, 073106 (2015); 10.1063/1.4928725

Nanomanufacturing of titania interfaces with controlled structural and functional properties by supersonic cluster beam deposition

Journal of Applied Physics 118, 234309 (2015); 10.1063/1.4937549

Cluster beam synthesis of nanostructured thin films

Journal of Vacuum Science \& Technology A: Vacuum, Surfaces, and Films 19, 2025 (2001); 10.1116/1.1331289

Resistive memory effects in $\mathrm{BiFeO}_{3}$ single crystals controlled by transverse electric fields

Applied Physics Letters 108, 162903 (2016); 10.1063/1.4947467

A simple scanning spectrometer based on a stretchable elastomeric reflective grating

Applied Physics Letters 104, 061910 (2014); 10.1063/1.4865427

\section{COMSOL}

\section{CONFERENCE}

\section{BOSTON}

OCTOBER 3-5

Boston Marriott Newton

Register Now

aCOMSOL 


\title{
High-throughput shadow mask printing of passive electrical components on paper by supersonic cluster beam deposition
}

\author{
Francesco Caruso, Andrea Bellacicca, and Paolo Milani ${ }^{\mathrm{a})}$ \\ CIMaINa and Dipartimento di Fisica, Università degli Studi di Milano, Via Celoria 16, 20133 Milano, Italy
}

(Received 16 February 2016; accepted 8 April 2016; published online 20 April 2016)

\begin{abstract}
We report the rapid prototyping of passive electrical components (resistors and capacitors) on plain paper by an additive and parallel technology consisting of supersonic cluster beam deposition (SCBD) coupled with shadow mask printing. Cluster-assembled films have a growth mechanism substantially different from that of atom-assembled ones providing the possibility of a fine tuning of their electrical conduction properties around the percolative conduction threshold. Exploiting the precise control on cluster beam intensity and shape typical of SCBD, we produced, in a one-step process, batches of resistors with resistance values spanning a range of two orders of magnitude. Parallel plate capacitors with paper as the dielectric medium were also produced with capacitance in the range of tens of picofarads. Compared to standard deposition technologies, SCBD allows for a very efficient use of raw materials and the rapid production of components with different shape and dimensions while controlling independently the electrical characteristics. Discrete electrical components produced by SCBD are very robust against deformation and bending, and they can be easily assembled to build circuits with desired characteristics. The availability of large batches of these components enables the rapid and cheap prototyping and integration of electrical components on paper as building blocks of more complex systems. Published by AIP Publishing.
\end{abstract}

[http://dx.doi.org/10.1063/1.4947281]

The fabrication of electronic circuits and microfluidic devices on paper is considered a very promising solution for the realization of low-cost disposable analytical devices and biodegradable green electronics. ${ }^{1-3}$ Recently, the interest for the use of paper as a substrate for foldable electronic components such as antennas and actuators has substantially increased in view of the realization of the so-called origami electronics, ${ }^{4,5}$ self-foldable kirigami soft robots, ${ }^{6}$ and power portable emergency locator transmitters based on origami microbial fuel cells. ${ }^{3}$

These applications rely on the so-called "chip-on-flex technologies" where electronic components have typical dimension of tens or hundreds of microns so miniaturization is not a critical issue. ${ }^{1}$ In this case, substrates must be flexible and foldable, and manufacturing methods should be cheap, easily scalable, compatible with rapid prototyping in order to produce batches of components to be integrated in functional systems. $^{1,2}$

The use of paper as a substrate is thus very effective for the fabrication of flexible and foldable electronic circuits. Its low cost and availability is ideal for rapid prototyping, it can be easily shaped and trimmed with scissors or cutters, and it can be used for complex self-standing 3D structures, fluidics, and electrochemical applications. ${ }^{3-7}$

Printing on paper relies on a technological tradition dating back from almost two thousand years and originating from China; it became a mass-producing technology in Europe from the fifteenth century. ${ }^{7}$ The direct printing of electronic components with various degrees of complexity on paper has taken inspiration from this tradition, and it is

\footnotetext{
${ }^{\text {a) }}$ Author to whom correspondence should be addressed. Electronic mail: pmilani@mi.infn.it
}

based on techniques such as flexography, offset, and screen printing. ${ }^{7}$ The fabrication of elements such as transistors, ${ }^{8}$ solar cells, ${ }^{9}$ and diodes; and devices such as displays, RadioFrequency IDentification (RFID) tags, and sensors is highly demanding in terms of the characteristics of the substrate. For a large number of applications plastic-covered paper is used and traditional solid-state circuital elements are glued on paper substrates. ${ }^{7}$ The printing of passive circuital elements on plain paper, such as resistors and capacitors, is less demanding; however, chip-on-flex and soft robotics technologies require the production of large batches of components to be subsequently assembled in $2 \mathrm{D}$ or $3 \mathrm{D}$ configurations, with different electrical and geometrical characteristics. ${ }^{1,6,10}$

Among different approaches, ink-jet printing is considered a technique of choice for the printing of simple electronic circuits on paper with the use of conductive inks and for the fabrication of passive and active elements. ${ }^{11}$ The use of ink-jet printing is limited by the availability of conducting inks with suitable properties (surface tension, viscosity) able to match the roughness, porosity, and wettability of the paper substrates; moreover, the post-printing ink drying process is difficult to control, and it can lead to unwanted variations of the electrical properties of the components. ${ }^{7}$

An alternative to the use of liquid inks has been developed in the last decade consisting in the direct writing with gas-phase coating techniques where an atomic vapor or nanoparticles dispersed in a carrier gas are deposited directly or through a shadow mask. ${ }^{12}$ Direct writing from the gas phase is a low-cost and high-throughput technology suitable also for 3D substrates, ${ }^{13}$ its widespread application is, however, hampered by the lack of acceptable adhesiveness on the substrate and electrical conductivity of the deposited species. $^{14}$ 
In general, traditional printing techniques have only a partial control on the amount of deposited conductive material; hence, as in the case of resistors, the value for the resistance is determined only by the length and the lateral dimension of the printed trace.

In the case of capacitors, the literature reports about interdigitated capacitors printed on plastic foils or photographic paper in a narrow range of low-capacitance values. ${ }^{7}$ The use of paper as dielectric layer between two printed metallic layers is an alternative solution as the rolling of metallized paper foils into cylinders is widespread for the fabrication of standard capacitors; however, no reports about this fabrication strategy are available.

We have demonstrated that the use of intense and highly collimated neutral nanoparticle beams produced in a supersonic expansion is an enabling tool for the large-scale integration of nanoparticles and nanostructured films on microfabricated platforms and smart nanocomposites. ${ }^{15-17}$ In particular, we have shown that this approach called supersonic cluster beam deposition (SCBD) can be efficiently used for the fabrication of electrically conductive patterned structures and devices on a wide variety of substrates, including polymers. ${ }^{18,19}$

Here we present the rapid and reproducible parallel fabrication of sets of resistors and capacitors on paper by using SCBD and a shadow mask. Our approach relies on the fact that the electrical properties of cluster-assembled films grown from the gas phase evolve in a significantly different way compared to atom-assembled films. ${ }^{20}$ In particular, in the percolative regime, electrical conduction can be precisely controlled by $\mathrm{SCBD},{ }^{21}$ thus allowing the tuning of the resistance while keeping constant the dimensions of the resistors and optimizing the use of raw material.

Figure 1(a) shows a schematic representation of the SCBD deposition apparatus equipped with a Pulsed Microplasma Cluster Source (PMCS). ${ }^{22}$ A PMCS consists in a ceramic body with a cavity where a metallic target (Au in the present case) is sputtered by a localized electrical discharge ignited during the pulsed injection of Ar gas at high pressure (40 bar). The sputtered metal atoms from the target thermalize with the carrier gas and aggregate in the cavity forming metal clusters. The carrier gas-cluster mixture expands out of the PMCS through a nozzle into a low pressure $\left(10^{-6} \mathrm{mbar}\right)$ expansion chamber (Fig. 1(a)). The supersonic expansion originating from the high pressure difference between the PMCS and the expansion chamber results in highly collimated supersonic beam: a divergence lower than $1^{\circ}$ is obtained by using aerodynamic focusing nozzles. ${ }^{22}$

The central part of the cluster beam enters a second vacuum chamber (deposition chamber, at a pressure of about $10^{-5} \mathrm{mbar}$ ), and it impinges on the shadow mask-substrate paper system supported by a motorized substrate holder. During deposition, the holder displaces the mask and the substrate in the vertical direction orthogonal to the cluster beam axis (Fig. 1(b)). Since the cluster density distribution in the beam is bell-shaped (as characterized in detail in Ref. 23), this results in a homogeneous metallization in the direction parallel to the raster with a gradient in the dose of nanoparticle in the direction perpendicular to the raster. This allows the one-step fabrication of components with different resistances in a controlled range of values. As a substrate, we used commercial plain white paper for ink-jet and laser printer (Xerox Digital, $75 \mathrm{~g} / \mathrm{m}^{2}$, roughness: $170 \mathrm{ml} / \mathrm{min}$ ) and the shadow mask was cut from another sheet of paper. The deposition area was of $50 \mathrm{~cm}^{2}$.

Figure 1(c) shows a photograph of a set of resistors printed on paper: the lateral dimensions of the resistors and their resistance are independent since the amount of clusters deposited to form each individual component can be controlled independently in an interval determined by the beam shape and the number of rastering cycles.

Figure 2(a) reports a scanning electron microscope (SEM) micrograph of the border region between metallized and pristine paper: the fibrous structure of the paper is evident. Figure 2(b) shows the border of a resistor deposited by SCBD with a step lateral resolution below $1 \mu \mathrm{m}$.

The typical deposition process for the production of twenty-seven resistors as shown in Fig. 1(c) takes roughly
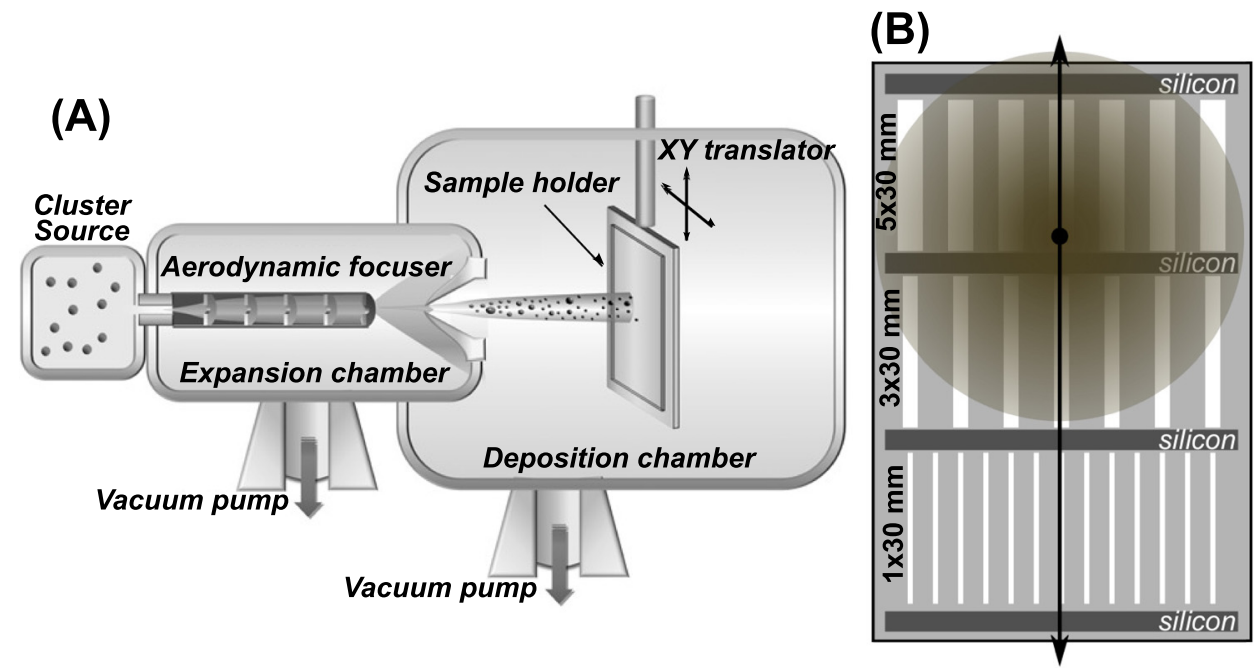

(C)

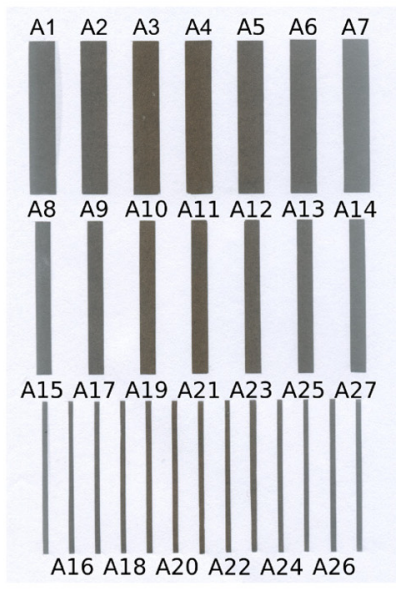

FIG. 1. (a) Schematic representation (not to scale) of a supersonic cluster beam deposition apparatus. (b) Shadow mask for resistor printing, the dark spherical area is the cluster beam deposition spot. The substrate and the mask are displaced along the vertical direction during deposition. (c) Photograph of a set of resistors printed in a one-step process with gold nanoparticles on plain paper. 

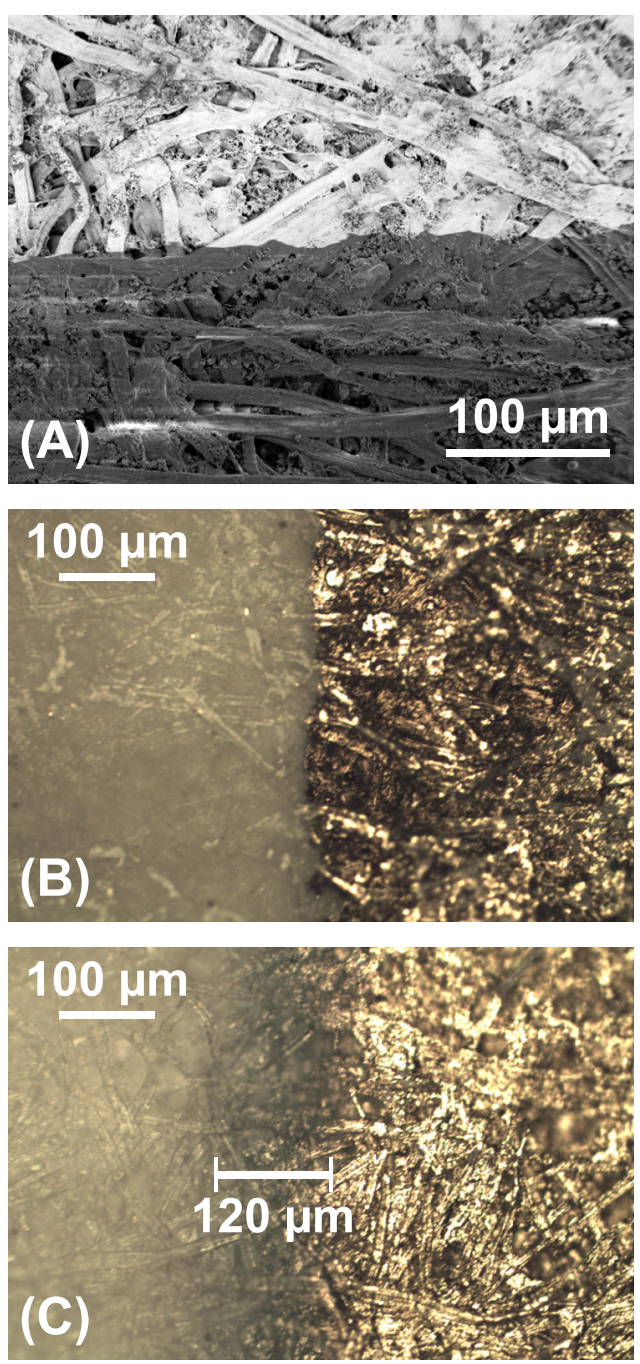

FIG. 2. (a) SEM micrograph of the border between plain paper (dark region) and the metallized one (white region). (b) Optical microscope micrograph of a border of a SCBD printed resistor. The step lateral resolution is below $1 \mu \mathrm{m}$. (c) Optical microscope micrograph of a border of evaporated resistor. The step lateral resolution is about $120 \mu \mathrm{m}$.

$30 \mathrm{~min}$, as a proof of principle we printed two sets of resistors ranging from $100 \Omega$ to $28 \mathrm{k} \Omega$ and $270 \Omega$ to $160 \mathrm{k} \Omega$, respectively. The resistance measurement was performed using a digital ohmmeter pressing two flat probes on the resistor short edges.

For the sake of comparison, we produced, using the same shadow mask, a set of resistors using gold evaporated by joule heating in a standard metallization apparatus. Gold was evaporated for about 10 min from a molybdenum crucible placed at a distance of roughly $30 \mathrm{~cm}$ from the substrate within a chamber at a pressure of about $5 \times 10^{-5} \mathrm{mbar}$.

The effusion rate from the crucible is angle dependent, ${ }^{24}$ and this, in principle, can be used to produce resistors with a different amount of material and hence different resistances. With this method, we obtained a set of resistors similar to that produced by SCBD, while in a considerably narrower resistance interval from 23 to $470 \Omega$. The use of a standard evaporator results also in lower lateral resolution: Fig. 2(c) shows the border of a resistor placed near the shadow mask margin (where the mask-substrate separation is lower), the measured lateral resolution is about $120 \mu \mathrm{m}$, and for a resistor in the middle of the shadow mask the resolution is over
$300 \mu \mathrm{m}$. Another disadvantage is that in the evaporative deposition method, a large quantity of gold is wasted in the chamber.

The growth mechanisms and the subsequent evolution of the electrical properties of cluster-assembled films are different from those of atom-assembled films. ${ }^{20,21}$ This indicates that the capability of SCBD of producing in one step resistors with different dimensions in a wide range of resistance values, is due to the capability of working in a well determined region of the electrical percolation curve of the cluster-assembled films..$^{21,23}$

In order to quantify the amount of clusters deposited on the substrate, we use a quantity called equivalent thickness $\left(\mathrm{t}_{\mathrm{eq}}\right)$ defined as the thickness of a film produced by an equivalent amount of nanoparticles deposited onto a rigid flat substrate. ${ }^{23}$ The equivalent thickness is obtained in real time, during the deposition process, by the use of a quartz crystal microbalance placed near the sample, allowing us to stop the deposition at the achievement of the required thickness and hence resistance. We checked $t_{\text {eq }}$ by measuring with an atomic force microscope the thickness of the cluster-assembled film grown on a silicon substrate mounted aside the paper substrate during deposition.

In order to compare the electrical properties of different components of the batches, the electrical sheet resistance $\left(R_{\mathrm{s}}\right)$ can be calculated for each resistor knowing its resistance and dimensions $\left(\mathrm{R}_{\mathrm{s}}=\mathrm{R}\right.$ - width/length); this quantity is independent of the resistor geometry and only varies with the amount of deposited clusters. Fig. 3(a) reports the electrical sheet resistances obtained for the two sets of resistors produced by $\mathrm{SCBD}$, as a function of equivalent thickness of the clusterassembled films. According to the percolation theory, ${ }^{25}$ we found that the trend is a power law $R_{s} \propto\left(t_{e q}-t_{c}\right)^{-t}$. Curve fitting was performed by weighted least square linear regression of $\ln \left(t_{\text {eq }}-t_{c}\right)$ on $\ln R_{s}$, where $t_{c}$ was manually changed
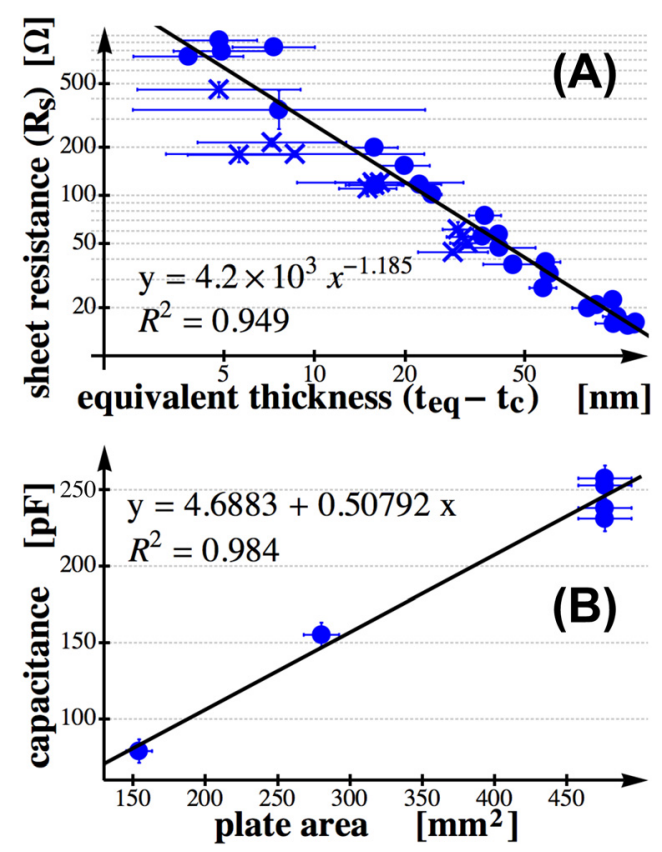

FIG. 3. (a) Electrical sheet resistance of cluster-assembled resistors on paper as a function of equivalent thickness. Curve fitting was performed by weighted least square linear regression of $\ln \left(\mathbf{t}_{\mathbf{e q}}-\mathbf{t}_{\mathbf{c}}\right)$ on $\ln \mathbf{R}_{\mathbf{s}}$. (b) Electrical capacitance of paper capacitors as a function of plate area. 
to maximize the coefficient of determination $\mathrm{R}^{2}$ (the uncertainty on $t_{c}$ was estimate considering the interval in which the $R^{2}$ does not change appreciably). The critical thickness $\left(t_{c}\right)$ of the cluster-assembled film above which we observe electrical conductance is $t_{c}=18 \pm 1 \mathrm{~nm}$, and the critical exponent of the percolation curve is $\mathrm{t}=1.185 \pm 0.043$ in good agreement with the theory $(1<\mathrm{t}<1.3)$.

Using this percolation curve, we are thus able to print a set of resistors around the exact value of any required resistance, keeping constant the dimensions of the single element. It should be noted that we are working in a percolative regime so the amount of raw material used to fabricate the resistors does not scale with the resistance values. This is very important since it shows that working in this regime with SCBD allows to minimize the amount of raw material while covering a wide range of resistance values for any resistor shape it may be useful for further applications.

With SCBD and a rectangular stencil mask, we also produced different capacitors printing the gold layer on both sides of the paper, thus obtaining a parallel plate capacitor with paper as the dielectric medium. We printed several capacitors varying the amount of deposited gold and the geometrical dimensions of the plates. As expected, the capacitance was independent of the quantity of deposited gold, and it was linear on the area of the conductive plate. From the trend line in the capacitance versus area plot, we obtain that the printed capacitance is of $50 \mathrm{pF} \mathrm{cm}^{-2}$ (Fig. 3(b)). Knowing the thickness of the paper used $(94 \pm 1 \mu \mathrm{m})$, we get a relative dielectric constant of $5.53 \pm 0.16$, close to the dielectric constant value of paper found in literature. ${ }^{26}$

Resistors and capacitors printed on paper by SCBD are very robust, and they are not affected by film delamination of cracking even under substantial bending and deformations. As a proof of principle of the easiness of combination of different paper components, we trimmed by scissors various resistors and capacitors, and we used them to assemble simple circuits such as RC frequency filters. We used clips to connect the different components.

In order to characterize the frequency response of a typical RC filter, we used a waveform generator to send a pure harmonic wave on the filter input and a digital oscilloscope to measure the amplitude of the input and output waveform. In Fig. 4, we report the Bode plots for a low pass and high pass typical RC filters obtained by using a resistor of $16.5 \mathrm{k} \Omega$ and a capacitor of $231 \mathrm{pF}$. Experimental data show that the $\mathrm{RC}$ circuit is stable and reliable over a wide range of frequencies.

In summary, we demonstrated the parallel fabrication of batches of passive electrical components (resistors and capacitors) on plain paper by supersonic cluster beam deposition. SCBD is an additive technique allowing the deposition of neutral metal nanoparticles with a high degree of collimation and low kinetic energy. Compared with traditional direct writing techniques, SCBD can be efficiently coupled with a shadow mask for the deposition of components with controlled structure, dimensions, and electrical properties on thermolabile substrates, while maintaining a high throughput production and a low raw metal consumption. Thanks to the fine control on the amount of deposited clusters, the resistance of the components produced by SCBD can be tuned working
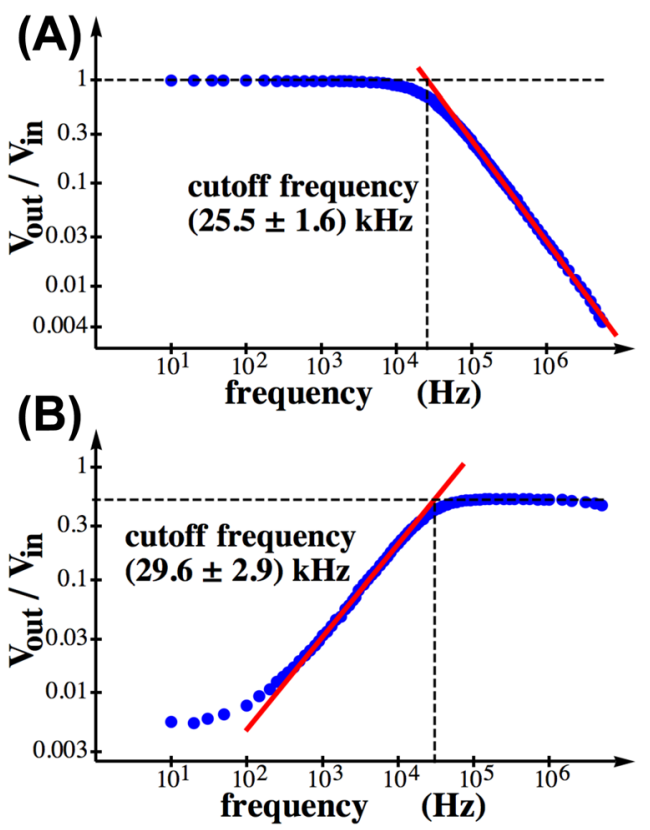

FIG. 4. Bode plots for RC filters using paper resistors and capacitors $(\mathrm{R}=16.5 \pm 0.2 \mathrm{k} \Omega, \mathrm{C}=231 \pm 8 \mathrm{pF})$.

in a suitable region of the percolation curve so that the dimension and the geometry of the resistor can be varied at will. This constitutes a substantial advantage compared to other deposition techniques such as thermal evaporation, ink jet printing and aerosol direct printing in terms of flexibility, rapidity of fabrication, optimization of the use of raw materials, and decoupling of components shape from electrical performances.

Simple circuits on paper can be easily assembled using the elements produced by SCBD; this opens the way to the rapid and cheap prototyping and integration of electrical components on paper as building blocks of more sophisticated systems for paper-based mechatronics.

${ }^{1}$ A. C. Siegel, S. T. Phillips, M. D. Dickey, N. Lu, Z. Suo, and G. M. Whitesides, Adv. Funct. Mater. 20, 28 (2010).

${ }^{2}$ R. F. P. Martins, I. M. M. Ferreira, and E. E. M. C. Fortunato, Phys. Status Solidi RRL 5, 332 (2011).

${ }^{3}$ J. Winfield, L. D. Chambers, J. Rossiter, J. Greenmanc, and I. Ieropoulos, J. Mater. Chem. A 3, 7058 (2015).

${ }^{4}$ M. Nogi, N. Komoda, K. Otsuka, and K. Suganuma, Nanoscale 5, 4395 (2013).

${ }^{5}$ P. K. Yang, Z. H. Lin, K. C. Pradel, L. Lin, X. Li, X. Wen, J. H. He, and Z. L. Wang, ACS Nano 9, 901 (2015).

${ }^{6} \mathrm{H}$. Shigemune, S. Maeda, Y. Hara, U. Koike, and S. Hashimoto, in Proceedings of the IEEE/RSJ Int. Conf. on Intelligent Robots and Systems (IROS) (2015), p. 1091.

${ }^{7}$ D. Tobjörk and R. Österbacka, Adv. Mater. 23, 1935 (2011).

${ }^{8}$ R. F. P. Martins, A. Ahnood, N. Correia, L. M. N. P. Pereira, R. Barros, P. M. C. B. Barquinha, R. Costa, I. M. M. Ferreira, A. Nathan, and E. E. M. C. Fortunato, Adv. Funct. Mater. 23, 2153 (2013).

${ }^{9}$ H. Águas, T. Mateus, A. Vicente, D. Gaspar, M. J. Mendes, W. A. Schmidt, L. Pereira, E. E. M. C. Fortunato, and R. F. P. Martins, Adv. Funct. Mater. 25, 3592 (2015).

${ }^{10}$ X. Liao, Q. Liao, X. Yan, Q. Liang, H. Si, M. Li, H. Wu, S. Cao, and Y. Zhang, Adv. Funct. Mater. 25, 2395 (2015).

${ }^{11}$ Y. Zheng, Z. He, Y. Gao, and J. Liu, Sci. Rep. 3, 1786 (2013).

${ }^{12}$ J. M. Hoey, A. Lutfurakhmanov, D. L. Schulz, and I. S. Akhatov, J. Nanotechnol. 2012, 324380 (2012).

${ }^{13}$ A. Mahajan, C. D. Frisbie, and L. F. Francis, ACS Appl. Mater. Interfaces 5, 4856 (2013). 
${ }^{14}$ N. S. Kim and K. N. Han, J. Appl. Phys. 108, 102801 (2010).

${ }^{15}$ E. Barborini, S. Vinati, M. Leccardi, P. Repetto, G. Bertolini, O. Rorato, L. Lorenzelli, M. Decarli, V. Guarnieri, C. Ducati, and P. Milani, J. Micromech. Microeng. 18, 055015 (2008).

${ }^{16}$ M. Marelli, G. Divitini, C. Collini, L. Ravagnan, G. Corbelli, C. Ghisleri, A. Gianfelice, C. Lenardi, P. Milani, and L. Lorenzelli, J. Micromech. Microeng. 21, 045013 (2011).

${ }^{17}$ T. Mazza, E. Barborini, I. N. Kholmanov, P. Piseri, G. Bongiorno, S. Vinati, P. Milani, C. Ducati, D. Cattaneo, A. Li Bassi, C. E. Bottani, A. M. Taurino, and P. Siciliano, Appl. Phys. Lett. 87, 103108 (2005).

${ }^{18}$ G. Corbelli, C. Ghisleri, M. Marelli, P. Milani, and L. Ravagnan, Adv. Mater. 23, 4504 (2011).

${ }^{19}$ C. Minnai and P. Milani, Appl. Phys. Lett. 107, 073106 (2015).
${ }^{20}$ J. Schmelzer, Jr., S. A. Brown, A. Wurl, M. Hyslop, and R. J. Blaikie, Phys. Rev. Lett. 88, 226802 (2002).

${ }^{21}$ E. Barborini, G. Corbelli, G. Bertolini, P. Repetto, M. Leccardi, S. Vinati, and P. Milani, New J. Phys. 12, 073001 (2010).

${ }^{22}$ P. Piseri, H. V. Tafreshi, and P. Milani, Curr. Opin. Solid State Mater. Sci. 8, 195 (2004).

${ }^{23}$ C. Ghisleri, F. Borghi, L. Ravagnan, A. Podestà, C. Melis, L. Colombo, and P. Milani, J. Phys. D: Appl. Phys. 47, 015301 (2014).

${ }^{24}$ M. Ohring, Materials Science of Thin Films, 2nd ed. (Academic Press, New York, 2002).

${ }^{25}$ S. Kirkpatrick, Rev. Mod. Phys. 45, 574 (1973).

${ }^{26}$ S. Simula, S. Ikäläinen, K. Niskanen, T. Varpula, H. Seppä, and A. Paukku, J. Imaging Sci. Technol. 43, 472 (1999). 